\title{
Effect of powdered seed of Nigella sativa administration on sub-chronic and chronic lead acetate induced hemato-biochemical and histopathological changes in Sprague Dawley rats
}

\begin{abstract}
Lead acetate $(\mathrm{PbAC})$ toxicity can occur by either ingestion or inhalation from contaminated surfaces or from the environment. Nigella sativa is a natural product with immense pharmacological properties, which include antioxidant, antibacterial, and antianemia properties. It has been showed to counter the effect of PbAC-induced hematological and biochemical changes in short-term studies. This study hypothesized that the $N$. sativa (NS) administration will ameliorate the deleterious effects of chronic PbAC toxicity in rats. A total of 75 Sprague Dawley rats were divided into three groups of 25 rats, and each group was further sub-divided into five groups of five rats each. Group 1 rats (negative control) were given distilled water, group 2 (positive control; PC) were given $10 \mathrm{mg} / \mathrm{kg}$ of lead acetate (PbAC) daily, and groups 3 (T1), 4 (T2), and 5 (T3) were each given $10 \mathrm{mg} / \mathrm{kg}$ of PbAC followed by graded concentrations of powdered seeds of NS; 100, 150, and $200 \mathrm{mg} / \mathrm{kg}$, respectively. Five rats in each group were euthanized at 30,60, and 90 days for collection of whole blood and selected organs. Whole blood was collected after euthanized via cardio puncture and used to evaluate the complete blood profile, while plasma was used for biochemical analysis. Tissue samples of the liver and kidney were fixed with $10 \%$ buffered formalin, processed, and stained with $H \& E$ and periodic acid-Schiff (PAS) for the liver. Aggression and fear were increased in the PbAC-exposed group and absent in the T3 group. There was a lower $(p<0.05)$ red blood cell count $(\mathrm{RBC})$, packed cell volume $(\mathrm{PCV})$, mean corpuscular hemoglobin concentration (MCHC), and lymphocyte count in the PC and T1 groups only. Biochemical analysis revealed elevated $(p<0.05)$ liver enzyme and creatinine levels in the PC and T1 groups on day 90 for AST and day 30 for ALT and creatinine. The level of alkaline phosphatase (ALP) was higher $(p<0.05)$ in the PC at 30 and 60 days of sampling. Other parameters, such as WBCs, prothrombin, urea, and cholesterol, were not significant in all groups. Histopathological lesions in the liver and kidneys were more severe in the PC and T1 groups, while the T2 and T3 groups showed mild lesions resulting from $N$. sativa administration. There was a decrease $(p<0.05)$ in the total PAS-stained area signifying glycogen depletion in the PC, T1, and T2 groups at 60 days and a higher distribution of the PAS-stained areas $(p<0.05)$ in the T3 group. At 90 days, the PC group had a lower $(p<0.05)$ distribution of PAS-stained areas in comparison to the other groups. The results showed the therapeutic potential of $N$. sativa extract in modulating both hematological and biochemical alterations induced by chronic lead acetate administration in rats.
\end{abstract}

Keyword: Nigella sativa; Lead acetate; Hematology; Biochemistry; Histopathology; Sprague Dawley rats 